\title{
The role of exportin 6 in cytoskeletal-mediated cell death and cell adhesion in human non-small-cell lung carcinoma cells following doxorubicin treatment
}

\author{
Magdalena Izdebska $^{1 \dagger}$, Maciej Gagat ${ }^{1 \dagger}$, Dariusz Grzanka ${ }^{2}$, Marta Halas ${ }^{1}$, Alina Grzanka ${ }^{1}$ \\ ${ }^{1}$ Department of Histology and Embryology, Nicolaus Copernicus University in Torun, \\ Collegium Medicum in Bydgoszcz, Bydgoszcz, Poland \\ ${ }^{2}$ Department and Clinic of Dermatology, Sexually Transmitted Diseases and Immunodermatology, \\ Nicolaus Copernicus University in Torun, Collegium Medicum in Bydgoszcz, Bydgoszcz, Poland \\ †These authors contributed equally to this work
}

\begin{abstract}
The actin cytoskeleton plays an important role in various cellular processes. The different forms of actin (G-actin and F-actin) participate in the organization of nuclear structure and its functions. The structure of the actin cytoskeleton is controlled by proteins involved in the translocation of actin between cytoplasm and the nucleus. In this study, we used siRNA method to investigate the role of exportin 6 in the switching between nuclear and cytoplasmic F-actin pools in $\mathrm{H} 1299$ cells treated with no, 1.0 or $2.5 \mu \mathrm{M}$ doxorubicin. We showed that silencing of exportin 6 expression changed the response of H1299 to doxorubicin. Here, we observed increased population of cells affected by doxorubicin-induced necrotic cell death. Furthermore, fluorescence studies showed that downregulation of exportin 6 exerted profound DOX-induced changes in the F-actin cytoskeleton architecture. The F-actin cytoskeleton was seen in the form of small fibers or aggregates after doxorubicin treatment. Additionally, some cells lost cell adhesion properties. Downregulation of exportin 6 influenced also transcriptional activity of the cells. In cells transfected with nontargeting siRNA, we observed a higher level of 5 'fluorouridine fluorescence than in cells with silenced exportin 6 expression. In conclusion, we showed that downregulation of exportin 6 induced necrotic cell death. Moreover, the observed alterations of cell adhesion suggest the key role of cytoplasmic F-actin in maintaining intercellular junctional complexes and/or focal adhesion properties and the importance of the balance between nuclear and cytoplasmic F-actin pools. (Folia Histochemica et Cytobiologica 2014, Vol. 52, No. 3, 195-205)
\end{abstract}

Key words: H1299 cells; F-actin; exportin 6; siRNA; doxorubicin; cell death; necrosis; fluorescence microscopy

\section{Introduction}

In recent years, many papers concerning the actin cytoskeleton have been published. It is well known that actin is localized in the cytoplasm and plays a key role in many cellular processes, such as proliferation, diffe-

\footnotetext{
Correspondence address: Prof. A. Grzanka Department of Histology and Embryology Nicolaus Copernicus University in Torun Collegium Medicum in Bydgoszcz

24 Karłowicza St., 85-092 Bydgoszcz, Poland tel.: +48525853725, fax: +48525853734 e-mail: agrzanka@cm.umk.pl
}

rentiation, and cell death. Actin filaments also participate in the control of cell shape, intracellular transport, cell motility, exocytosis, endocytosis, and carcinogenesis [1-3]. The cytoplasmic localization of actin is important in maintaining not only cell homeostasis, but also its level in the cell nucleus. Nuclear actin was identified for the first time over 30 years ago, but its functional significance and form have been questioned for a long time. The study presented by Dingová et al. showed that the nuclear actin structure is fundamentally different from actin structures observed in the cytoplasm, and pointed out that the equilibrium between nuclear actin forms is controlled and 
regulated by cytoplasmic actin-binding proteins [4]. It was also postulated that many different forms of actin are involved in organizing nuclear structure and function. It has now been established that nuclear actin is associated with all three RNA polymerases (Pol I, II, III) and is a component of many chromatin remodeling complexes, such as mammalian BAF complex, which belongs to the family of SWI/SWF-like chromatin-remodeling complexes [5-7]. Percipalle et al. demonstrated that actin colocalized with the heterogeneous nuclear ribonucleoprotein hrp65-2 in Chironomus tentants, which was important in the transcriptional activity of cells $[8,9]$. Moreover, there is evidence that actin has also the ability to bind to hrp36 and to participate in the process of transcription of Balbiani rings by RNA polymerase (RNAP II) $[8,9]$. Furthermore, actin was localized in the nucleoli and it was suggested that nuclear actin cooperates with nuclear myosin and takes part in the transport of ribosomal subunits to the cytoplasm [10]. Actin was also found in Cajal bodies, which suggests their role in the transport of ribonuclear particles (RNPs) and small RNAs [11-13].

The mechanisms of the nucleocytoplasmic transport of actin tend to be very complex on account of the size of actin and because of the lack of a classical nuclear localization signal (NLS) [14]. It has also been suggested that many actin-binding proteins have NLS, which thus makes it possible to import actin into the nucleus [15]. In 2003, a novel family of proteins, exportin 6 (XPO6), was identified. It was shown that exportin 6 enabled the translocation of actin to the cytoplasm in a functional complex with profiling [16]. During translocation, the actin is connected with exportin 6, but the profilin is needed to enhance their interactions [16].

Due to the previous studies that have indicated the significance of nuclear actin and/or nuclear F-actin in active cell death types, including apoptosis and mitotic catastrophe, we have decided here to investigate the effects of doxorubicin (DOX) in the adenocarcinoma cell line H1299 with an alternated balance between nuclear and cytoplasmic actin pools [2-3].

The aim of the present study was to determine the effects of downregulating the expression of exportin 6 on the sensitivity of human non-small-cell lung carcinoma H1299 cells to the cytostatic effect of DOX.

\section{Material and methods}

Cell culture and treatment. The H1299 cell line (human non-small-cell lung carcinoma cells; NSCLC) was purchased from the American Type Culture Collection. The cells, grown in Tissue Culture Flasks $\left(25 \mathrm{~cm}^{2}\right)$ or 12 -well plates
(BD Biosciences, Franklin Lakes, NJ, USA), were cultured in a monolayer in RPMI 1640 medium (Lonza, Verviers, Belgium) supplemented with $10 \%$ fetal bovine serum (FBS; Gibco/Invitrogen Life Technologies, Carlsbad, CA, USA) and $50 \mu \mathrm{g} / \mathrm{mL}$ gentamycin (Sigma-Aldrich, St. Louis, MO, USA) $\left(37^{\circ} \mathrm{C}\right.$ and $\left.5 \% \mathrm{CO}_{2}\right)$.

To induce cell death, the H1299 cells were treated with doxorubicin (DOX; Sigma-Aldrich) diluted in 1X DPBS (Dulbecco's Phosphate-Buffered Saline; Sigma-Aldrich) at concentrations of 1 and $2.5 \mu \mathrm{M}$ for $24 \mathrm{~h}$. The control cells were grown under the same conditions but without the addition of DOX.

Transfection method. To transfect H1299 cells, the cells were grown up to 80-90\% confluency in RPMI 1640 (Lonza) with FBS (Gibco/Life Technologies) and $50 \mu \mathrm{g} / \mathrm{mL}$ gentamycin (Sigma-Aldrich). Following trypsinization, the suspended cells were transfected using the SE Cell Line 4D-Nucleofector X Kit (Lonza), according to the manufacturer's instructions. Briefly, $1 \times 10^{6}$ cells were suspended in $100 \mu \mathrm{l}$ of SE nucleofector solution mixed with siRNA Hs_XPO6_1, predesigned to be directed against human XPO6 (Qiagen, Hilden, Germany). Next, the mixture of cells and transfection solution was transferred into transfection cuvettes and electroporated using a 4D-Nucleofector system (Lonza). To determine the nonspecific effects of siRNA transfection, nontargeting AllStars negative control siRNA (Qiagen) was used. At $72 \mathrm{~h}$ post transfection, the cells were used for further experiments. Each experiment was repeated independently three times.

Western blot analysis. Semiquantitative analysis of the posttranslational expression of exportin 6 was performed using the Western blot technique, according to the method described by Kong et al. with some modifications [17]. Briefly, the cells were lysed with RIPA buffer (Sigma-Aldrich). Following, normalization of the protein concentration was performed using a BCA protein assay kit (Thermo Scientific Pierce, Rockford, IL, USA). Equal amounts of protein $(15 \mu \mathrm{g}$ of total protein per lane) were separated using 4-12\% NuPage Bis-Tris gel (Novex/Life Technologies, Carlsbad, CA, USA) and transferred onto nitrocellulose membranes using the iBlot dry western blotting system (Invitrogen/Life Technologies). Prestained molecular weight markers (Thermo Scientific Pierce) were used to estimate the position of the protein bands. Subsequently, the membranes were processed using the BenchPro 4100 card processing station (Invitrogen/ /Life Technologies, Carlsbad, CA, USA). The membranes were blocked with $5 \%$ nonfat milk in TBS-T for $2 \mathrm{~h}$, and then incubated with the primary rabbit anti-exportin 6 (1:200; Santa Cruz Biotechnology, Dallas, TX, USA) and rabbit anti-GADPH (1:5,000; Sigma-Aldrich) antibodies, diluted in TBS-T for $2 \mathrm{~h}$ at room temperature. After washing with TBS-T, the membranes were incubated with the secondary 
antibodies conjugated with peroxidase (1:80,000; Sigma-Aldrich), diluted in TBS-T for $1 \mathrm{~h}$ at room temperature. The immunoreactive bands were visualized using enhanced chemiluminescence (ECL) on CL-XPosure Film (Thermo Scientific Pierce). After scanning, the densitometry of the bands was quantified using Quantity One Basic software, v. 3.6.5 (Bio-Rad; Hercules, CA, USA).

Analysis of cell death. The analysis of cell death was carried out using a Tali image-based cytometer (Invitrogen/Life Technologies) and a Tali apoptosis kit (Invitrogen/Life Technologies), according to manufacturer's instructions. After incubation of the cells with 1 or $2.5 \mu \mathrm{M}$ DOX, the cells were trypsinized, centrifuged (300 g for $5 \mathrm{~min}$ ), and resuspended in $100 \mu \mathrm{L}$ Annexin binding buffer. After that, $5 \mu \mathrm{L}$ of Annexin V Alexa Fluor 488 (Invitrogen/Life Technologies) was added to each sample, mixed well, and incubated at room temperature in the dark for $20 \mathrm{~min}$. Next, the cells were centrifuged and resuspended again in $100 \mu \mathrm{L}$ of Annexin binding buffer. Then, $1 \mu \mathrm{L}$ of propidium iodine (PI) was added to each sample, and samples were incubated at room temperature in the dark for $3 \mathrm{~min}$. The analysis was performed using a Tali image-based cytometer and the FCS Express Research Edition software (v. 4.03; De Novo Software, Glendale, CA, USA) on the condition that early apoptotic cells stain only green with Annexin V, late apoptotic cells stains with both PI (blue) and Annexin V, necrotic cells stain with PI, and live cells remain unstained.

Fluorescence microscopy. Staining of exportin 6/F-actin. The H1299 cells transfected with siRNA ${ }_{\mathrm{XPO} 6}$ and nontargeting siRNA were seeded on sterile glass coverslips placed in 12-well plates (BD Biosciences) and stained according to method described by Kinikoglu et al. (2014), with some modifications [18]. After $24 \mathrm{~h}$, the cells were treated with either $1 \mu \mathrm{M}$ or $2.5 \mu \mathrm{M}$ DOX for additional $24 \mathrm{~h}$ and then fixed with $4 \%$ paraformaldehyde in PBS for $20 \mathrm{~min}$ at room temperature. Next, the cells were washed with PBS $(3 \times 5$ min), permeabilized with $0.1 \%$ Triton $X-100$ in PBS for 5 min, washed again with PBA, and blocked in $1 \%(\mathrm{w} / \mathrm{v}) \mathrm{BSA} /$ /PBS for $1 \mathrm{~h}$. Exportin 6 was stained with rabbit anti-exportin 6 antibody (1:100; Santa Cruz Biotechnology) and then with anti-rabbit antibody-Alexa Fluor 555 (1:500; Invitrogen/ /Life Technologies). The cells were then incubated with phalloidin conjugated to Alexa Fluor 488 (1:40; Invitrogen/ /Life Technologies) for $20 \mathrm{~min}$ at room temperature. Nuclear staining was performed with 4',6'-diamidino-2-phenylindole dihydrochloride (100 ng/mL; DAPI, Sigma-Aldrich) for $10 \mathrm{~min}$. All incubations were performed at room temperature. After counterstaining with DAPI, the cells were rinsed with PBS and mounted in Aqua-Poly/Mount (Polysciences, Warrington, PA, USA). The cells were examined using a C1 laser-scanning confocal microscope (Nikon, Tokyo, Japan). 5 FUrd/F-actin staining. After the DOX treatment, the
H1299 cells were incubated in complete medium containing 5'-fluorouridine (FUrd) (Sigma-Aldrich), for $20 \mathrm{~min}$ at $37^{\circ} \mathrm{C}$ in $5 \% \mathrm{CO}_{2}$, fixed with $4 \%$ paraformaldehyde in PBS for $20 \mathrm{~min}$ at room temperature and permeabilized with $0.1 \%$ Triton X-100 in PBS for 5 min. The cells were then blocked with $1 \%$ BSA and stained with mouse anti-BrdU (1:250; Sigma-Aldrich) in PBS to visualize the incorporated FUrd. After $1 \mathrm{~h}$ incubation with the primary antibody, the cells were washed with PBS and stained for $1 \mathrm{~h}$ using anti-mouse secondary antibody conjugated to Alexa Fluor 488 (Invitrogen/Life Technologies). Next, the cells were washed and incubated with phalloidin conjugated to TRITC (Sigma-Aldrich) for $20 \mathrm{~min}$. Nuclear staining was performed with DAPI (100 ng/mL; Sigma-Aldrich) for $10 \mathrm{~min}$. All incubations were performed at room temperature. After counterstaining, the cells were rinsed with PBS and mounted in Aqua-Poly/Mount (Polysciences). The cells were examined using a classical fluorescence and C1 laser-scanning confocal microscopes (Nikon, Tokyo, Japan).

Statistical analysis. The statistical analyses were performed with the Mann-Whitney $U$-test using GraphPad Prism software (Ver5.0; GraphPad software). The results were considered significant at $p \leq 0.05$.

\section{Results}

\section{Downregulation of exportin 6 by the siRNA}

The downregulation of exportin 6 expression with siRNAHs_XPO6_1, predesigned to be directed against human XPO6, was examined by using the Western blotting method. The densitometric analysis indicated a significant reduction in the posttranslational expression of exportin 6 in the siRNA-transfected control cells exposed to 1 and $2.5 \mu \mathrm{M}$ DOX (Figure 1). After

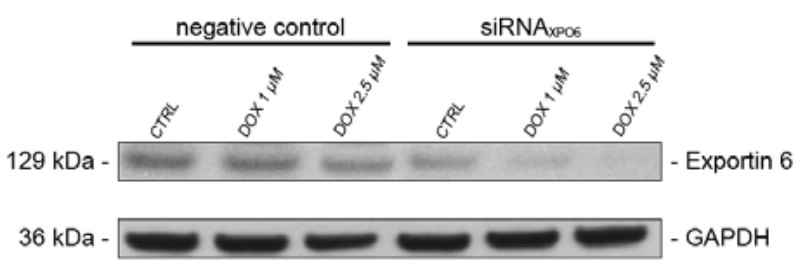

Figure 1. Silencing of exportin 6 expression. H1299 cells were transfected with nontargeting AllStars negative control siRNA and siRNA Hs_XPO6_1 predesigned against human XPO6, exposed to $0,1.0$ and $2.5 \mu \mathrm{M}$ DOX. After treatment, exportin 6 proteins were analyzed by Western blot. After transfection of H1299 cells with siRNA, the silencing of exportin 6 expression was $64.36 \%, 89.80 \%$, and $94.28 \%$, compared to control and 1 or $2.5 \mu \mathrm{M}$ DOX-treated cells, respectively. Abbreviations: CTRL — control cells; DOX - doxorubicin-treated cells 


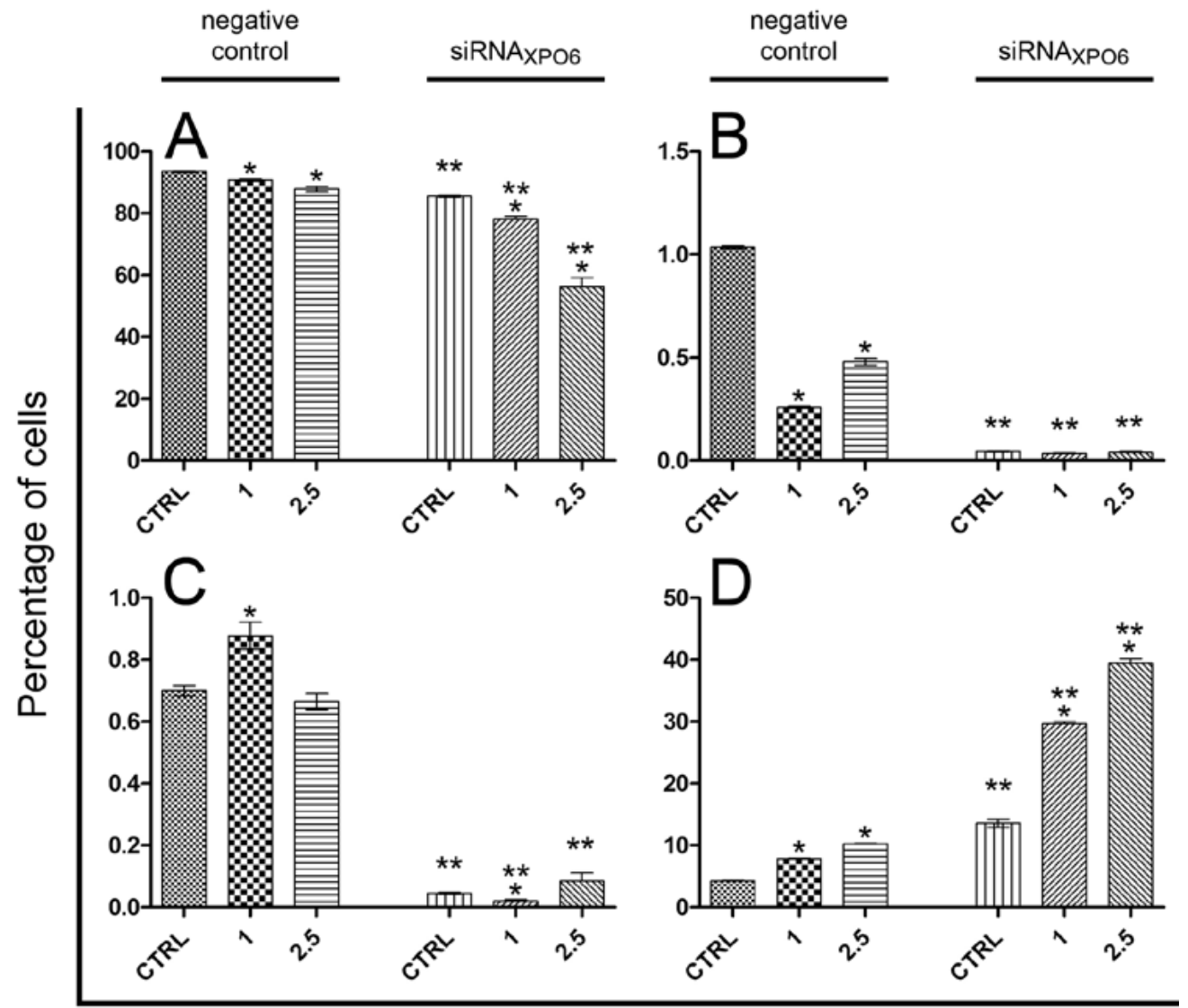

Doxorubicin concentration

Figure 2. Effect of DOX on the induction of cell death. H1299 transfected with the siRNA negative control and siRNA Hs_XPO6_1 were treated with $0,1.0$ and $2.5 \mu \mathrm{M}$ doxorubicin for $24 \mathrm{~h}$ and double-stained with Annexin V Alexa Fluor 488 and propidium iodide. Analysis was performed using an image-based cytometer as described in Methods. A. Percentage of live cells in $\mathrm{H} 1299$ cells transfected with siRNA negative control and siRNA ${ }_{\mathrm{XPO}}$; B. Percentage of early apoptotic cells; C. Percentage of late apoptotic cells; D. Percentage of necrotic cells. *Statistically significant differences between control cells and cells treated with doxorubicin; $p<0.05$; Mann-Whitney $U$-test. ** Statistically significant differences between cells transfected with siRNA negative control or siRNA knockdown of exportin 6; $p<0.05$; Mann-Whitney $U$-test

the transfection of the H1299 cells with siRNA, the level of exportin 6 was $64.36 \%, 89.80 \%$, and $94.28 \%$ compared to control cells transfected with nontargeting siRNA and treated with DOX at doses 1 and $2.5 \mu \mathrm{M}$, respectively (Figure 1 ).

\section{Downregulation of exportin 6 promotes DOX-induced necrosis}

Cell death analysis was performed using a Tali image-based cytometer and Tali apoptosis kit, consisting of Annexin V-Alexa Fluor 488 and propidium iodide to identify cells undergoing apoptotic and necrotic cell death.

In the cells transfected with nontargeting siRNA and siRNA ${ }_{\mathrm{XPO} O}$, statistically significant differences in the percentage of live cells were observed following treatment with all doses of DOX, as compared to the appropriate control cells. The percentage of live cells transfected with nontargeting siRNA and treated with 1 and $2.5 \mu \mathrm{M}$ DOX was slightly decreased from $93.4 \%$ to $90.7 \%(p=0.04)$ and $87.8 \%(p=0.04)$, respectively, in comparison to the untreated control cells (Figure 2A). Statistically significant differences in the percentage of live cells were noticed in siRNA ${ }_{\mathrm{XPO}}{ }^{-t r a n s f e c t e d}$ cells. The percentage of live control cells was $85.5 \%$, however, after treatment of the cells with 1 and $2.5 \mu \mathrm{M}$ DOX, it decreased to $78.2 \%(p=0.0361)$ and $56.2 \%$ $(p=0.0361)$, respectively (Figure 2A). Statistically significant differences in the percentage of live cells between cells transfected with nontargeting siRNA and siRNA $_{\text {ХРО }}$ were also noticed (Figure $2 \mathrm{~A}$ ).

Moreover, we observed a small population of early apoptotic cells, stained only with Annexin V and of 
late apoptotic cells, stained with propidium iodide and Annexin V. In the H1299 cells transfected with non-targeting siRNA, a statistically significant decrease, from $1.03 \%$ to $0.26 \%$ and $0.48 \%$, in the percentage of early apoptotic cells was observed following treatment with 1 and $2.5 \mu \mathrm{M}$ DOX, respectively (Figure 2B). However, in the population of siRNA $\mathrm{XPO}^{-}$-transfected cells, statistically significant differences were not noticed. However, statistically significant decreases between cells transfected with nontargeting siRNA and siRNA ${ }_{\mathrm{XPO}}$ were observed (Figure 2B). The analysis of the DOX-induced late apoptosis in cells transfected with nontargeting siRNA showed a statistically significant increase in the percentage of cells, from $0.70 \%$ to $0.88 \%$, following treatment with $1 \mu \mathrm{M}$ DOX. In the case of siRNA ${ }_{\mathrm{XPO} 6}$-transfected cells, the statistically significant differences were observed only after treatment of cells with $1 \mu \mathrm{M}$ DOX (Figure 2C).

As shown in Figure 2D, the population of necrotic cells following transfection with nontargeting siRNA and treatment with DOX was statistically significant increased from $4.23 \%$ to $7.85 \%$, and $10.29 \%$ in the control and after treatment with doses of 1 and $2.5 \mu \mathrm{M}$ DOX, respectively. Moreover in siRNA ${ }_{\mathrm{XPO} 6}$-transfected cells, the differences between the control and the cells treated with DOX were highly significant: $13.7 \%$ of control cells were necrotic, and this value increased to $29.7 \%(p<0.0001)$ and $39.5 \%(p<0.0001)$ after treatment with 1 and $2.5 \mu \mathrm{M}$ DOX, respectively. Moreover, statistically significant differences between the cells transfected with nontargeting siRNA and siRNA $_{\mathrm{XPO} 6}$ were also noticed.

\section{Downregulation of exportin 6 expression disrupts F-actin cytoskeleton and reduces cell adhesion}

The morphology of the control cells transfected with nontargeting siRNA was not changed as compared to non-transfected cells: they adhered to each other, had a polyhedral shape, and contained one oval or kidney-shaped nucleus. In these cells, a voluminous network of actin cytoskeleton and bundles of tension fibers were observed. We also noticed short fibers of actin filaments in the perinuclear area and high intensity of cortical F-actin fluorescence in the cell-cell interaction areas (Figure 3A and $\mathrm{A}^{\prime}$ ). Exportin 6 was located in the cell nuclei (Figure $3 \mathrm{~A}$ and $\mathrm{A}^{\prime}$ ). Following treatment with DOX, the architecture of the actin cytoskeleton was changed. The dose of $1 \mu \mathrm{M}$ DOX induced a significantly lower number of stress fibers. F-actin appeared in the form of short fibers in the cytoplasm and in the perinuclear area. Nevertheless, contact between cells and the intense F-actin fluorescence in the cell-cell interactions were observed. Additionally, giant cells with multiple nuclei of different sizes were seen. In cells exposed to the $1 \mu \mathrm{M}$ dose of DOX, a higher intensity of exportin 6 in the nucleus was seen than in the control (Figure 3B and B'). The degradation of the F-actin cytoskeleton was observed following treatment of cells with $2.5 \mu \mathrm{M}$ DOX. Furthermore, the short fibers of the actin filaments in the cytoplasm, F-actin, aggregated in the perinuclear region, and the highest intensity of exportin 6 fluorescence was also seen there (Figure 3C and C').

The transfection of cells with siRNA ${ }_{\mathrm{XPO}}$ induced profound changes in the F-actin cytoskeleton. We observed a few cells on the slides that lost cell adhesion properties (Figure 4A'-C'). In the control cells, which were not incubated with DOX, thick stress fibers of F-actin were observed (Figure 4A). After treating the cells with $1 \mu \mathrm{M}$ DOX, the F-actin cytoskeleton was destroyed and only small fibers or F-actin aggregates were observed (Figure 4B). Similarly, the destruction of the actin cytoskeleton was seen following treatment with $2.5 \mu \mathrm{M}$ DOX (Figure 4C). However, the number of small fibers or F-actin aggregates was greater than in cells treated with $1 \mu \mathrm{M}$ DOX.

Additionally, in cells transfected with nontargeting siRNA, we observed a gradual increase in the fluorescence of the F-actin in the nuclear area following DOX treatment (Figure 3A-C'). Nuclear F-actin was also seen in siRNA ${ }_{\mathrm{XPO} 6}$-transfected cells in the control and in cells treated with DOX (Figure 4A-C'). However, the nuclear F-actin accumulation was more evident and associated with weaker DNA staining.

\section{Downregulation of exportin 6 induces changes in the transcriptional activity of the H1299 cells}

The transcriptional activity of cells was assessed using a 5-fluorouridine (FUrd) incorporation assay. In cells transfected with nontargeting siRNA, we observed a higher level of 5-FUrd fluorescence than in the siRNA ${ }_{\text {Хос6 }}$-transfected cells (Figures 5A-C and $6 \mathrm{~A}-\mathrm{C}$ ). All the nuclei of the controls transfected with nontargeting siRNA were characterized by very intense fluorescence of 5-FUrd, but after treatment with DOX, this signal gradually decreased. We also noticed that in morphologically altered nuclei, the intensity of the 5-FUrd was lower (Figure 5A-C). In the control of siRNA $\mathrm{XPO}$-transfected cells, the intensity of the 5-FUrd was very high, but foci-like signals were seen (Figure 6A). The similar results were seen in cells with multiple nuclei following treatment with $1 \mu \mathrm{M}$ DOX (Figure 6B). By contrast, in $2.5 \mu \mathrm{M}$ DOX treated cells, the identification of 5-FUrd was not possible (Figure 6C). The structure 


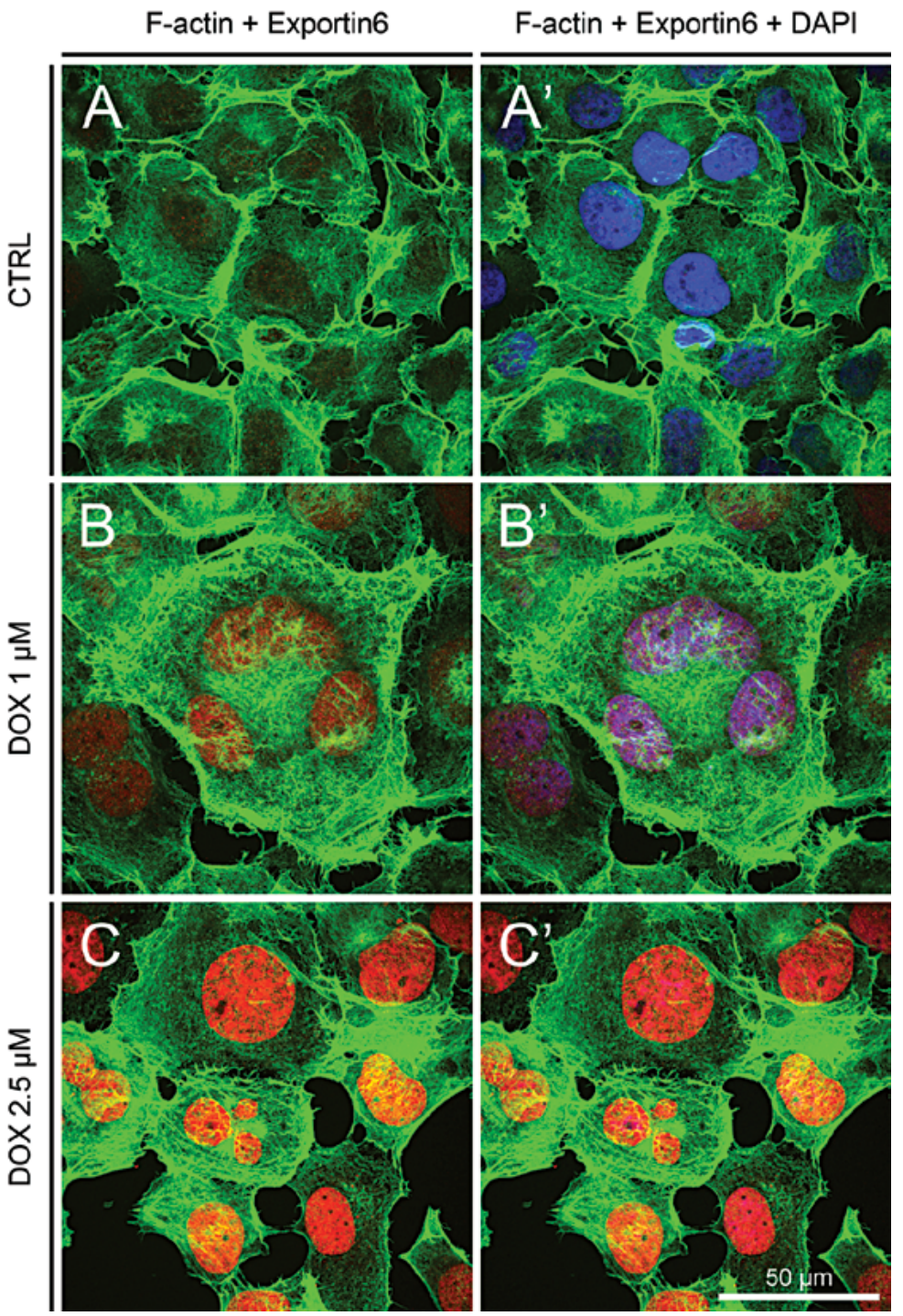

Figure 3. Localization of exportin 6 and F-actin in control and DOX-treated H1299 cells. H1299 cells were treated with $0,1.0$ and $2.5 \mu \mathrm{M}$ DOX and labeled for the presence of exportin 6 (red) and F-actin (green) as described in Methods. Cell nuclei were labeled with DAPI. A-A'. Control cells: very low nuclear fluorescence signal of exportin 6 and typical for H1299 cells architecture of F-actin cytoskeleton; B-B'. $1 \mu \mathrm{M}$ DOX: nuclear fluorescence of exportin 6 and F-actin in the form of short fibers in the cytoplasm and in the perinuclear area; C-C'. $2.5 \mu \mathrm{M}$ DOX: high nuclear fluorescence signal of exportin 6 and degradation of the F-actin cytoskeleton. Abbreviations as for Figure 1. Bar, $50 \mu \mathrm{m}$

of the actin cytoskeleton was identical to that described above (Figures 5A'-C' and 6A'-C'). Moreover, in both negative controls and in the cells transfected with siRNA ${ }_{\mathrm{XPO}}$, weaker DNA staining was observed following DOX treatment.

\section{Discussion}

Changes in nuclear actin levels have been linked to various cellular processes, because actin may function as a switch between transcriptional states. Actin has neither a classic nuclear localization signal (NLS) nor a specific import receptor, so the transport of this protein is hampered. However, many actin-binding proteins contain a nuclear localization sequence and can thus be responsible for the translocation of actin into the nucleus [15].

It is known that cofilin is one such protein mediating the active transport of actin into nucleus under physiological conditions; as an actin-binding protein (ABP), it plays a role in the regulation of actin dynamics [19]. We previously reported that downregulation of cofilin influenced changes in the localization pool of F-actin in CHOAA8, and as a consequence, 


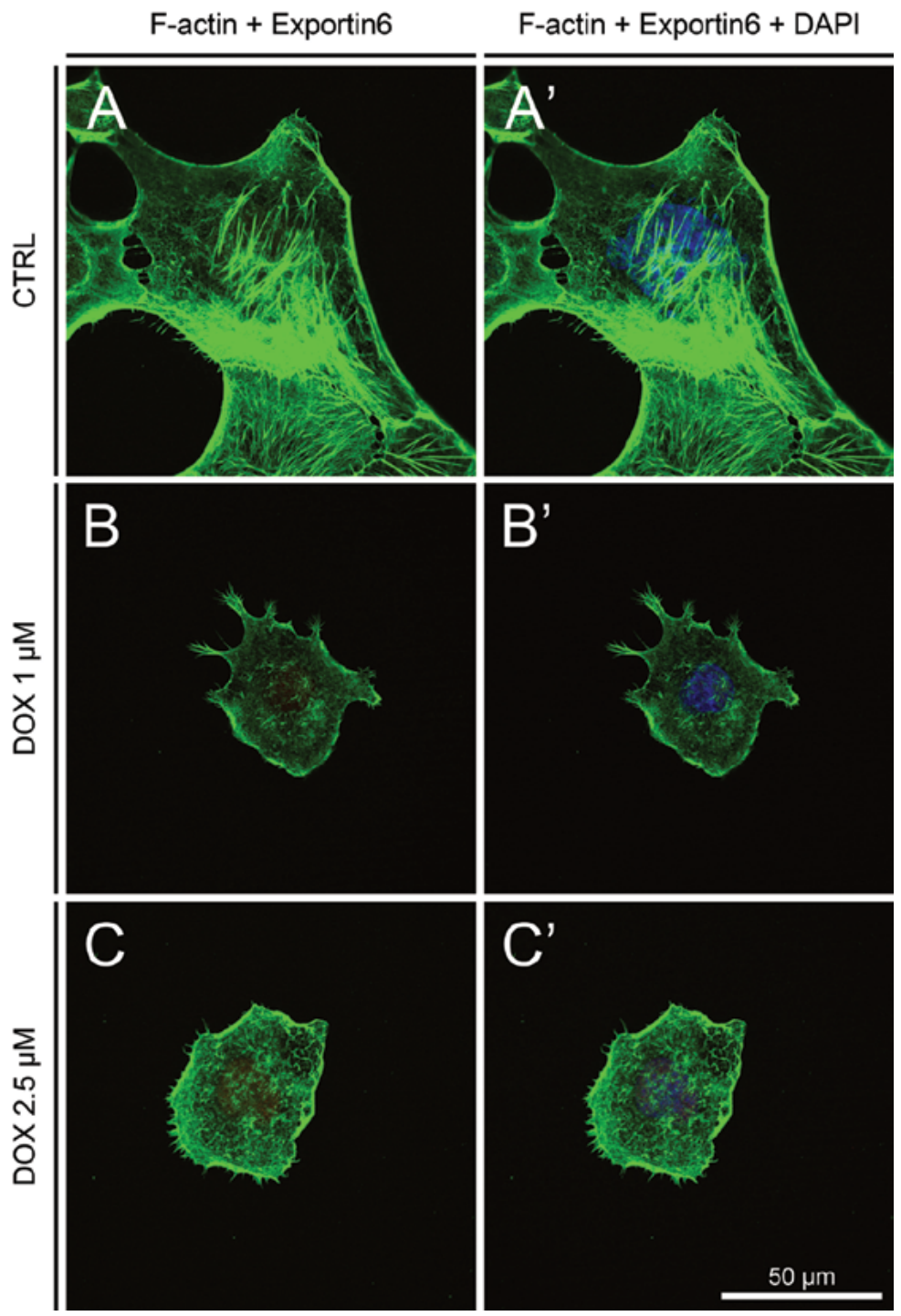

Figure 4. Localization of exportin 6 and F-actin in control and DOX-treated H1299 cells with silenced XPO6 expression. H1299 cells transfected with siRNA ${ }_{\mathrm{XPO} 6}$ were treated with $0,1.0$ and $2.5 \mu \mathrm{M}$ DOX and labeled for the presence of exportin 6 (red) and F-actin (green) as described in Methods. Cell nuclei were labeled with DAPI. A-A'. Control cells: lack of exportin 6 fluorescence signal and perturbation of F-actin cytoskeleton architecture; B-B'. $1 \mu$ M DOX: lack of exportin 6 fluorescence signal and destruction of F-actin; C-C'. $2.5 \mu \mathrm{M}$ DOX: lack of exportin 6 fluorescence signal and destruction of F-actin. Abbreviations as for Figure 1. Bar, $50 \mu \mathrm{m}$

no apoptotic cell death was observed following treatment with DOX [20-21]. These results were very interesting, because they presented the important role of nuclear F-actin in cell-death induction processes. Therefore, in this study we have examined the effect of downregulating the expression of exportin 6 , another important protein in the translocation of F-actin between the cell nucleus and the cytoplasm, in the context of the reorganization of the actin cytoskeleton and also of the sensitivity of human non-small-cell lung carcinoma cells (H1299 cell line) to DOX which is a well-known cytostatic drug.
We observed changes in the organization of actin filaments and the various fluorescent intensities of exportin 6 in cells transfected with siRNA ${ }_{\mathrm{XPO}}$. The results also showed that silencing of exportin 6 changed not only form and distribution of actin cytoskeleton but also the adhesion properties of cells. At both applied DOX concentrations, there was a significant reduction in the number of stress fibers, similarly to the findings of Pawlik et al. on H1299 cells after treatment with phenethyl isothiocyanate [22]. We also observed the presence of F-actin in the nucleus and its higher fluorescence after DOX treatment. 

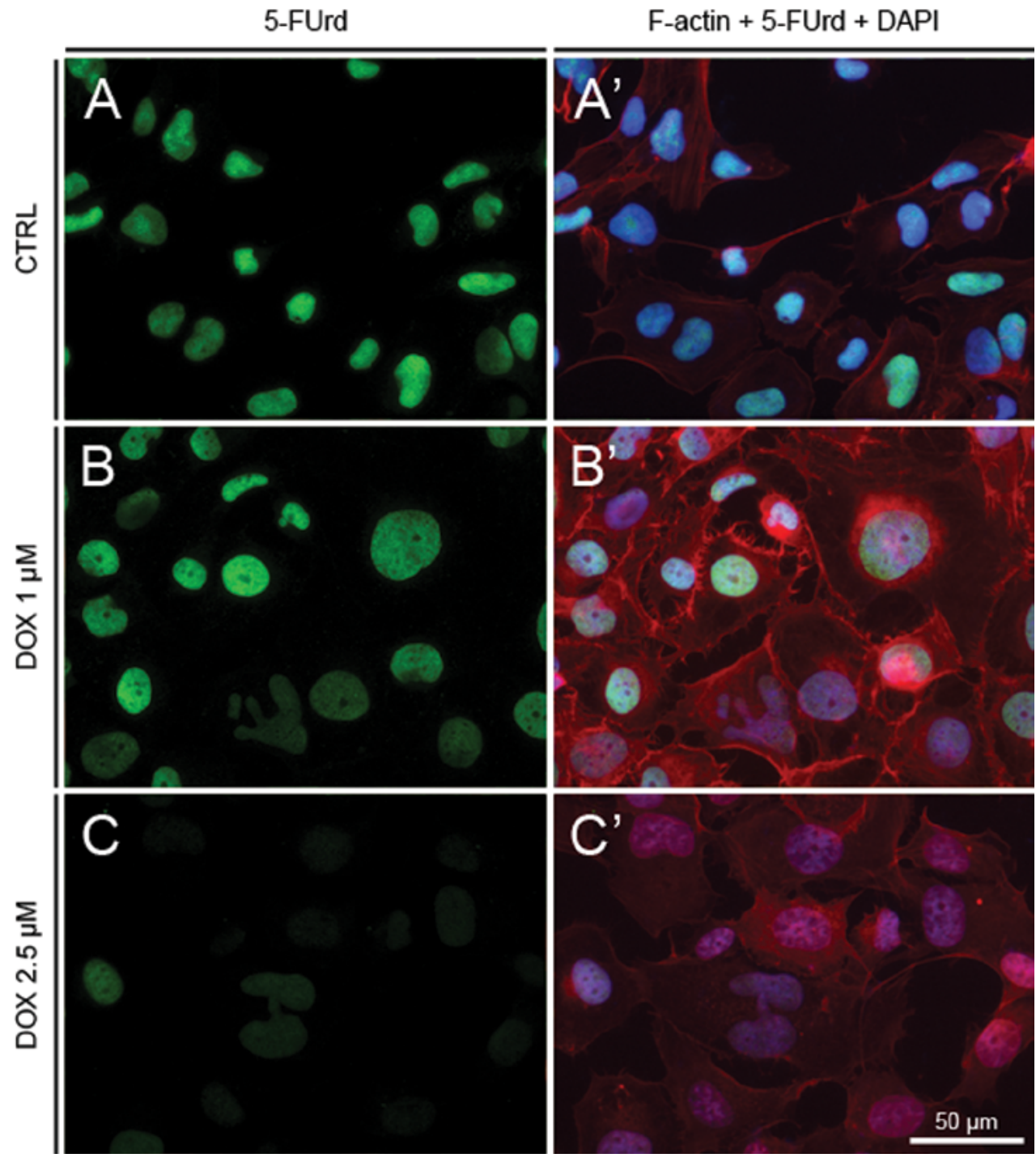

Figure 5. Effect of DOX on transcriptional activity of the H1299 cells. H1299 cells were treated with $0,1.0$ and $2.5 \mu \mathrm{M}$ doxorubicin and labeled for the presence of 5-FUrd (green) and F-actin (red) as described in Methods. Cell nuclei were labeled with DAPI. A-A'. Control cells; B-B'. $1 \mu \mathrm{M}$ DOX; C-C'. $2.5 \mu \mathrm{M}$ DOX. Abbreviations as for Figure 1 and 5-FUrd -5'-fluorouridine. Bar, $50 \mu \mathrm{m}$

However, it has been shown here that the DNA staining and transcriptionally active regions in the cell nucleus were reduced. On the other hand, Johnson et al. (2013) observed the enhancement of the nuclear localization of actin when cells were exposed to stress conditions, and suggested that nuclear F-actin is involved in structural changes of chromatin during cell death or the transcription of genes significant for cell death induction [23]. However, the normal H1299 cells are resistant to DOX due to the lack of functional expression of p53 [24] so that cell death was thus not observed after treatment with this cytostatic drug [25]. However, after the H1299 cells transfected with siRNA ${ }_{\mathrm{XPO} 6}$ were treated with DOX, we observed the induction of cell death.
It is known that the mechanism of DOX action is highly dose-dependent. Many studies using different cell lines showed that low concentrations of DOX promote cell senescence or mitotic catastrophe, while higher doses induce apoptosis [26-29]. In the present study, H1299 cells showed cell shape changes following DOX treatment. In siRNA-negative control transfected cells which had been treated with DOX, we observed a few giant cells with lobulated nuclei or multiple nuclei, characteristic of mitotic catastrophe. The mechanism of this type of cell death is not clear, but the described process is usually combined with abnormal mitosis and multinucleated giant cells [30-32]. Results similar to those of the present paper were presented by Pawlik et al. on hyperthermia tre- 


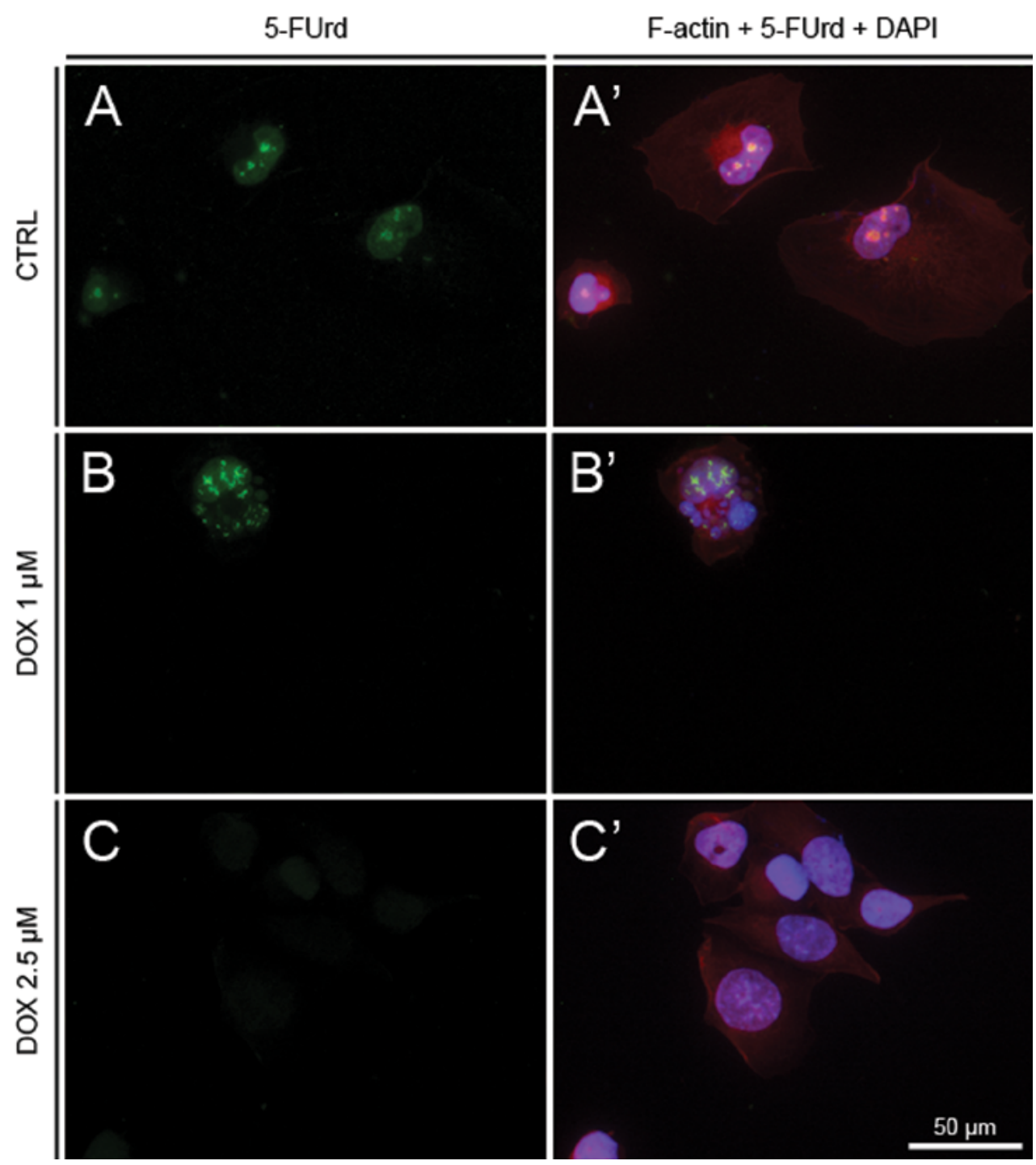

Figure 6. Effect of DOX on transcriptional activity of the H1299 cells with silenced XPO6 expression. H1299 cells transfected with siRNA ${ }_{\mathrm{XPO}}$ were treated with $0,1.0$ and $2.5 \mu \mathrm{M}$ doxorubicin and labeled for the presence of 5-FUrd (green) and F-actin (red) as described in Methods. Cell nuclei were labeled with DAPI. A-A'. Control cells; B-B'. $1 \mu \mathrm{M}$ DOX; C-C'. 2.5 $\mu \mathrm{M}$ DOX. Abbreviations as for Figure 5. Bar, $50 \mu \mathrm{m}$

atment of H1299 cells [22], Zhang et al. on exposure of human lung cancer cells to 8-chloro-adenosine [33], and $\mathrm{Li}$ et al. using the adenovirus E4orf4 protein [34]. These reports all indicated that mitotic catastrophe frequently occurred in H1299 cells cultured under stress conditions [33-35]. Our image-based cytometry analysis of Annexin V/propidium iodide double-stained H1299 cells showed the externalization of phosphatidylserine in the plasma membrane, which is typical of apoptosis, but these populations of cells transfected with nontargeting siRNA and siRNA were very small. However, in this study, a dose-dependent increase and PI-double positive cells were observed. This is in agreement with the study of Ulu- kaya et al. who showed that various compounds (such as platinum and palladium complexes) did not induce apoptosis, but rather necrosis [36]. Similar results were presented by Li et al. who found that adenovirus E4orf4 protein-induced membrane blebbing resulted in the formation of multiple nuclei and increased cell volume, which are typical for necrosis [35]. We suggest here that this type of cell death is associated with the H1299 cell line treated with DOX. It has also been pointed out that mitotic catastrophe is induced in cells that lack functional p53 [37]. By contrast, as in the case of the H1299 cell line, Tang et al. have associated this mutation with necrosis, suggesting a novel link between p53 and inflammatory proces- 
ses [38]. However, in the present study, evidence for DOX-induced necrosis was noticed.

Our another major observation involved the alterations of cell shapes associated with the reorganization of the F-actin cytoskeleton. The actin maintains the cell's shape and participates in regular cellular motility [39]. These processes are highly dependent on the rearrangements of the F-actin cytoskeleton and the balance between cytoplasmic and nuclear actin pools. Dopie et al. confirmed that actin is constantly exported out of the nucleus and that this process is mediated by exportin 6, independently of Crm1 [40]. This contradicts the data presented by Stüven et al. that $\mathrm{Crm} 1$ plays important role in actin transport from mammalian or insect nuclei [16]. In our study, we used siRNA technique to downregulate the expression of exportin 6, and similarly to Bohnsack et al. [41] we showed an accumulation of actin in the nucleus after transfection with siRNA $\mathrm{XPO}_{\text {. }}$. Moreover, we observed that only a few cells remained on the slides following the transfection of the $\mathrm{H} 1299$ cells with siRNA which suggest that this may be connected with altered translocation of actin between cytoplasm and cell nucleus. This assumption is confirmed by our previous study, which demonstrated that the stabilization of the cytoplasmic pool of F-actin by the overexpression of tropomyosin-1 strengthens cellular junctions through the preservation of F-actin's polymerization state, and then protects endothelial cells against stress agents (such as L-homocysteine) [42].

Nuclear actin is a key regulator of polymerase II-dependent transcription and is required for RNA polymerase I transcription [43-44]. Zhang et al. showed the localization of FUrd in the nucleoli and connected this with active RNA polymerase I transcription [45]. On the other hand, Obrdlik and Percipalle observed the same localization of 5-FUrd as in the present study, and related this staining with polymerase II RNA. They also showed that cofilin-1 was involved in the machinery of transcription of certain genes, in which interaction with actin was involved [46]. Additionally, they suggested that the controlled polymerization of actin may be important for polymerase II transcription. In the present study, in the control of siRNA ${ }_{\mathrm{XOP}}$-transfected cells and in cells with multiple nuclei following treatment with $1 \mu \mathrm{M}$ DOX we noticed foci-like structures of 5-FUrd indicative of transcriptional activity. In contrast, in cells treated with $2.5 \mu \mathrm{M}$ DOX, the identification of 5-FUrd was not possible, what suggest the lack of transcription. In a similar way, Obrdlik and Percipalle showed very low levels of FUrd foci fluorescence after the silencing of the cofilin-1 gene [46]. They associated these changes with the strict relationship between the involvement of cofilin-1 in polymerase II transcription elongation and the polymerization of gene-associated actin. It is also very interesting that the low level of cofilin-1 expression was related to the increase in the nuclear F-actin pool, meaning that the silencing of the cofilin- 1 gene induced the polymerization of actin and promoted the formation of atypical F-actin structures in the nucleus area [46]. All the studies presented above show the different ways in which actin contributes in the transcription machinery. Our study provides further clues to the actin involvement in many processes inside the nucleus and suggests that exportin 6 alters the balance between nuclear and cytoplasmic pools of F-actin via the induction of different cell-death modes.

In conclusion, we showed that after downregulation of exportin 6 expression in H1299 cells, doxorubicin induced necrotic cell death. Also important was the observation of cell adhesion alterations, which support the key role of cytoplasmic F-actin in cell adhesion and the importance of maintaining of nuclear and cytoplasmic pools of F-actin.

\section{Acknowledgment}

The study was supported by grant No. MN-2/WL from Nicolaus Copernicus University (NCU) and by statutory activities no. 896.

\section{References}

1. Firat-Karalar EN, Welch MD. New mechanisms and functions of actin nucleation. Curr Opin Cell Biol. 2011;23:4-13.

2. Grzanka D, Gagat M, Izdebska M. Actin is required for cellular death. Acta Histochem. 2013;115:775-782.

3. Grzanka D, Gagat M, Izdebska M. Involvement of the SAT$\mathrm{B} 1 / \mathrm{F}$-actin complex in chromatin reorganization during active cell death. Int J Mol Med. 2014;33:1441-1450.

4. Dingová H, Fukalová J, Maninová M, Philimonenko VV, Hozák P. Ultrastructural localization of actin and actin-binding proteins in the nucleus. Histochem Cell Biol. 2009;131:425-434.

5. Rando OJ, Zhao K, Janmey P, Crabtree GR. Phosphatidylinositol-dependent actin filament binding by the SWI/SNF-like BAF chromatin remodeling complex. Proc Natl Acad Sci USA. 2002;99:2824-2829.

6. Zhao K, Wang W, Rando OJ et al. Rapid and phosphoinositol-dependent binding of the SWI/SNF-like BAF complex to chromatin after $\mathrm{T}$ lymphocyte receptor signaling. Cell. 1998;95:625-636.

7. Bogolyubova I. F-actin distribution pattern in the nuclei of early mouse embryos. Folia Histochem Cytobiol. 2009;47: 461-463.

8. Percipalle P, Fomproix N, Kylberg K et al. An actin-ribonucleoprotein interaction is involved in transcription by RNA polymerase II. Proc Natl Acad Sci USA. 2003;100: 6475-6480.

9. Percipalle P,ZhaoJ, Pope B, Weeds A, Lindberg U, Daneholt B. Actin bound to the heterogeneous nuclear ribonucleoprotein hrp36 is associated with Balbiani ring mRNA from the gene to polysomes. J Cell Biol. 2001;153:229-236. 
10. Cisterna B, Necchi D, Prosperi E, Biggiogera M. Small ribosomal subunits associate with nuclear myosin and actin in transit to the nuclear pores. FASEB J. 2006;20:1901-1903.

11. Cruz JR, Moreno Díaz de la Espina S. Subnuclear compartmentalization and function of actin and nuclear myosin I in plants. Chromosoma. 2009;118:193-207.

12. Dundr M, Ospina JK, Sung MH et al. Actin-dependent intranuclear repositioning of an active gene locus in vivo. $J$ Cell Biol. 2007;179:1095-1103.

13. Gedge LJ, Morrison EE, Blair GE, Walker JH. Nuclear actin is partially associated with Cajal bodies in human cells in culture and relocates to the nuclear periphery after infection of cells by adenovirus 5. Exp Cell Res. 2005;303:229-239.

14. Skarp KP, Vartiainen MK. Actin on DNA - an ancient and dynamic relationship. Cytoskeleton (Hoboken). 2010;67: 487-495.

15. Vartiainen MK. Nuclear actin dynamics-from form to function. FEBS Lett. 2008;582:2033-2040.

16. Stüven T, Hartmann E, Görlich D. Exportin 6: a novel nuclear export receptor that is specific for profiling actin complexes. EMBO J. 2003;22:5928-5940.

17. Kong Y, Grimaldi M, Curtin E et al. Neural crest development and craniofacial morphogenesis is coordinated by nitric oxide and histone acetylation. Chem Biol. 2014;21:488-501.

18. Kinikoglu B, Kong Y, Liao EC. Characterization of cultured multipotent zebrafish neural crest cells. Exp Biol Med (Maywood). 2014;239:159-168.

19. Bamburg JR. Proteins of the ADF/cofilin family: essential regulators of actin dynamics. Annu Rev Cell Dev Biol. 1999;15:185-230.

20. Grzanka D, Marszałek A, Gagat M, Izdebska M, Gackowska L, Grzanka A. Doxorubicin-induced F-actin reorganization in cofilin-1 (nonmuscle) down-regulated CHO AA8 cells. Folia Histochem Cytobiol. 2010;48:377-386.

21. Grzanka D, Marszałek A, Izdebska M, Gackowska L, Szczepanski MA, Grzanka A. Actin cytoskeleton reorganization correlates with cofilin nuclear expression and ultrastructural changes in CHO AA8 cell line after apoptosis and mitotic catastrophe induction by doxorubicin. Ultrastruct Pathol. 2011;35:130-138.

22. Pawlik A, Szczepanski MA, Klimaszewska A, Gackowska L, Zuryn A, Grzanka A. Phenethyl isothiocyanate-induced cytoskeletal changes and cell death in lung cancer cells. Food Chem Toxicol. 2012;50:3577-3594.

23. Johnson MA, Sharma M, Mok MT, Henderson BR. Stimulation of in vivo nuclear transport dynamics of actin and its co-factors IQGAP1 and Rac1 in response to DNA replication stress. Biochim Biophys Acta. 2013;1833:2334-2347.

24. Breen L, Heenan M, Amberger-Murphy V, Clynes M. Investigation of the role of $\mathrm{p} 53$ in chemotherapy resistance of lung cancer cell lines. Anticancer Res. 2007;27:1361-1364.

25. Mi J, Zhang X, Rabbani ZN et al. RNA aptamer-targeted Inhibition of NF-kappa B suppresses non-small cell lung cancer resistance to doxorubicin. Mol Ther. 2008;16:66-73.

26. Eom YW, Kim MA, Park SS et al. Two distinct modes of cell death induced by doxorubicin: apoptosis and cell death through mitotic catastrophe accompanied by senescence-like phenotype. Oncogene. 2005;24:4765-4777.

27. Litwiniec A, Grzanka A, Helmin-Basa A, Gackowska L Grzanka D. Features of senescence and cell death induced by doxorubicin in A549 cells: organization and level of selected cytoskeletal proteins. J Cancer Res Clin Oncol. 2010;136:717-736.
28. Park SS, Eom YW, Choi KS. Cdc2 and Cdk2 play critical roles in low dose doxorubicin-induced cell death through mitotic catastrophe but not in high dose doxorubicin-induced apoptosis. Biochem. Biophys. Res. Commun. 2005;334:1014-1021.

29. Park SS, Kim MA, Eom YW, Choi KS. Bcl-xL blocks high dose doxorubicin-induced apoptosis but not low dose doxorubicin-induced cell death through mitotic catastrophe. Biochem Biophys Res Commun. 2007;363:1044-1049.

30. Abend M, Gilbertz KP, Rhein A, van Beuningen D. Early and late $\mathrm{G} 2$ arrest of cells undergoing radiation-induced apoptosis or micronucleation. Cell Prolif. 1996;29:101-113.

31. Miranda EI, Santana C, Rojas E, Hernández S, Ostrosky-Wegman P, García-Carrancá A. Induced mitotic death of HeLa cells by abnormal expression of c-H-ras. Mutat Res. 1996;349:173-182.

32. Roninson IB, Broude EV, Chang BD. If not apoptosis, then what? Treatment-induced senescence and mitotic catastrophe in tumor cells. Drug Resist Updat. 2001;4:303-313.

33. Zhang HY, Gu YY, Li ZG et al. Exposure of human lung cancer cells to 8-chloro-adenosine induces $\mathrm{G} 2 / \mathrm{M}$ arrest and mitotic catastrophe. Neoplasia. 2004;6:802-812.

34. Li S, Szymborski A, Miron MJ et al. The adenovirus E4orf4 protein induces growth arrest and mitotic catastrophe in H1299 human lung carcinoma cells. Oncogene. 2009;28:390-400.

35. PawlikA, NowakJM, GrzankaD, GackowskaL, MichalkiewiczJ, Grzanka A. Hyperthermia induces cytoskeletal alterations and mitotic catastrophe in p53 deficient H1299 lung cancer cells. Acta Histochem. 2013;115:8-15.

36. Ulukaya E, Ari F, Dimas K et al. Cell death-inducing effect of novel palladium(II) and platinum(II) complexes on non-small cell lung cancer cells in vitro. J Cancer Res Clin Oncol. 2011;137:1425-1434.

37. Grzanka D, Stepien A, Grzanka A, Gackowska L, Helmin-Basa A, Szczepanski MA. Hyperthermia-induced reorganization of microtubules and microfilaments and cell killing in $\mathrm{CHO}$ AA8 cell line. Neoplasma. 2008;55:409-415.

38. Tang X, Molina M, Amar S. p53 short peptide (p53pep164) regulates lipopolysaccharide-induced tumor necrosis factor-alpha factor/cytokine expression. Cancer Res. 2007;67: 1308-1316.

39. Reymann AC, Boujemaa-Paterski R, Martiel JL et al. Actin network architecture can determine myosin motor activity. Science. 2012;336:1310-1314.

40. Dopie J,SkarpKP, RajakyläEK, TanhuanpääK, VartiainenMK. Active maintenance of nuclear actin by importin 9 supports transcription. Proc Natl Acad Sci USA. 2012;109:E544-E552.

41. Bohnsack MT, Stüven T, Kuhn C, Cordes VC, Görlich D. A selective block of nuclear actin export stabilizes the giant nuclei of Xenopus oocytes. Nat Cell Biol. 2006;8:257-263.

42. Gagat M, Grzanka D, Izdebska M, Grzanka A. Effect of L-homocysteine on endothelial cell-cell junctions following F-actin stabilization through tropomyosin-1 overexpression. Int J Mol Med. 2013;32:115-129.

43. Philimonenko VV, Zhao J, Iben $\mathrm{S}$ et al. Nuclear actin and myosin I are required for RNA polymerase I transcription. Nat Cell Biol. 2004;6:1165-1172.

44. Qi T, Tang W, Wang L, Zhai L, Guo L, Zeng X. G-actin participates in RNA polymerase II-dependent transcription elongation by recruiting positive transcription elongation factor b (P-TEFb). J Biol Chem. 2011;286:15 171-15 181.

45. Zhang Y, Forys JT, Miceli AP, Gwinn AS, Weber JD. Identification of DHX33 as a mediator of rRNA synthesis and cell growth. Mol Cell Biol. 2011;31:4676-4691.

46. Obrdlik A, Percipalle P. The F-actin severing protein cofilin-1 is required for RNA polymerase II transcription elongation. Nucleus. 2011;2:72-79. 